\title{
Clinical neuropsychiatric correlates and EEG findings among children with developmental disorders in Lagos, Nigeria
}

\author{
OF Aina', OC Ogun², HTO Ladapo ${ }^{2}$, FEA Lesi ${ }^{3}$, OO Famuyiwa' ${ }^{1}$ \\ 1Department of Psychiatry, ${ }^{3}$ Department of Paediatrics, College of Medicine, University of Lagos, Lagos, Nigeria \\ 2Psychiatric Hospital, Yaba, Lagos, Nigeria
}

\begin{abstract}
Objective: Developmental disorders with or without associated neuropsychiatric complications continue to be one of the major health problems in Africa. The grossly inadequate management/ rehabilitative facilities further worsen this. A prospective study aimed at finding the types of developmental disorders and associated neuropsychiatric complications among children aged $\leq 15$ years that presented with developmental disorder in the study centers over 36 month study duration. Methods: The study was carried out in the paediatric and child psychiatric clinics as well as the Electroencephalographic (EEG) unit of two major health facilities in Lagos, Nigeria: Lagos University Teaching Hospital (LUTH) and Psychiatric Hospital, Yaba, Lagos. For each subject, socio-demographic data was obtained and appropriate clinical evaluation was carried out to obtain the necessary data and clinical diagnoses. Furthermore, each of the subjects had waking EEG recording using 20-channel computerized Medelec® EEG machine. The EEG interpretation was blinded to the clinical history of the subjects. Results: Overall, one hundred and eleven (11 1) subjects were evaluated over the 36 month study period. The cohort was made up of 63 (56.8\%) males and $48(43.2 \%)$ females. The mean age was $4.8( \pm 3.9)$ years, with most subjects falling in the age group of 0-5 years(69.4\%). Mixed specific developmental disorders were most common (55\%) followed by that of specific developmental disorders of speech and language (34.2\%). Forty-one (36.9\%) subjects suffered from one or more types of complications, with seizure, 22(19.8\%) being the most common. The waking EEG recording was normal in 22 (19.8\%) subjects; while abnormal epileptiform activities were found in 85 (76.6\%) of recordings. No statistically significant relationship existed between EEG abnormalities and the factors of age and clinical diagnoses (i.e developmental abnormalities). Conclusion: The small number of subjects in this study is a major hindrance to drawing a general conclusion. However, it has been shown that a number of the cohort in addition to their developmental disorders suffered from such complications as seizures, hyperactivity etc. Furthermore, a significant proportion had EEG abnormalities of the epileptiform types possibly reinforcing the previously known fact of prevalent subtle brain damage among African children. The need for preventive health care is therefore emphasized.
\end{abstract}

Key Words: Developmental disorders; Associated complications; EEG abnormalities.

Received: $23-08-2007$

Accepted: 31-10-2007

\section{Introduction}

Behavioural and developmental disorders are the two broad groups of mental health problems in children. ${ }^{1}$ As end results of genetic aberrations, obstetric problems and/or postnatal insults, developmental disorders are quite common worldwide. According to the Centres for Diseases Control (CDC), developmental disorders are defined as "a diverse group of physical, cognitive, psychological, sensory and speech

\section{Correspondence:}

Dr OF Aina, Department of Psychiatry, College of Medicine

University of Lagos. PMB 12003, Lagos, Nigeria

email: ainafran@yahoo.com impairments that begin anytime during development up to 18 years of age". Consequently, this definition recognizes a wide range of conditions from psychomotor developmental delays or disorders to the more serious ones such as mental retardation, cerebral palsy and autism spectrum disorders. ${ }^{3}$ In contrast, some authors differentiated developmental disorders from cognitive impairment by submitting that disorders of development do not affect intellectual function; but when this is present, the condition is mental retardation. ${ }^{4}$

This present study focuses on psychomotor developmental delays or disorders; which are divided into specific disorders in which only a single function is involved (such as language or motor) and global developmental disorder in which a wide range 
of psychomotor developmental fields are affected. ${ }^{4}$ The specific developmental disorders are the commonest of developmental pathologies; and studies from advanced countries showed delayed speech or language development affects $5-10 \%$ of preschool children. For motor delays, it has been estimated that 2-3\% of infants fall outside the range of normal motor milestone attainment; and are of most concern to parents when their children are between ages 6-12 month. ${ }^{5}$

Given the structural and/or metabolic damage to the brain associated with most cases of developmental disorders, and the attending neuropsychiatric manifestations, this has resulted in a significant overlap in the presentation and management of most developmental and neuropsychiatric disorders. Studies among children from the West African subregion, which are few, show that 9.9\% of children referred for psychiatric evaluation have developmental delay. ${ }^{6}$ Despite the enormity of this problem and the prevalent causative factors of poor obstetric services and infections in developing countries ${ }^{7}$, the identification of developmental disorder is usually delayed. ${ }^{8}$ Aside from this delayed diagnosis, it has also been known that it is difficult most times to pick up specific causes of developmental disorders; however, they often result from a perinatal brain lesion that is not obvious enough to produce physical handicaps. ${ }^{9,10}$ Also of note is the role of EEG which has been criticized by some authors for its limited usefulness; notwithstanding this, electroencephalography (EEG) remains a valuable tool in the investigation of subtle cerebral damage not only among children with developmental disorders but in child psychiatric practice generally. ${ }^{11}$

To the best knowledge of the authors, no known published study has attempted to investigate the different clinical correlates and EEG abnormalities among children with developmental disorders in Nigeria. It is hoped that with the acquisition of modern digital EEG facilities in hospitals in the subregion, this study will improve the diagnostic capabilities among doctors who manage neuro-psychiatric and developmental disorders in the sub-region.

\section{Methods}

\section{Study Locations}

The study was carried out on subjects with developmental disorders from the following centres:

- The Neurology unit of the Dept. of Paediatrics, Lagos University Teaching Hospital (LUTH), Idi-Araba, Lagos.

- Dept. of Psychiatry, Lagos University Teaching Hospital, 6, Harvey Road (LUTH Annexe), Yaba, Lagos.

- Child and Adolescent Unit (Harvey Road Clinic), Psychiatric Hospital, Yaba, Lagos.

The Electroencephalographic recording on the subjects was at the EEG unit, Psychiatric Hospital, Yaba, Lagos; while the study secretariat was located at the Department of Psychiatry, (LUTH Annexe), Lagos.

The 2nd and 3rd study centres are neighbours atYaba area of Lagos; and over the years, there exists close collaboration in both clinical as well research activities between the two institutions.

\section{Subjects}

These included children aged $\leq 15$ years and diagnosed to have developmental disorders from history and clinical evaluation. Furthermore, they must have EEG recording done on them.

\section{Procedure}

The study took place from 1st January 2002 to 31 st December 2004. It was a prospective study on new consecutive paediatric subjects ( $\leq 15$ years) diagnosed to have developmental disorder from the study centres and who had an EEG recording conducted during the study period. For each of the subjects, appropriate socio-demographic data and clinical histories were obtained. Where necessary, further pre-recording interview was carried out in the EEG unit to fill missing gaps in the subject clinical history.

For nearly all the subjects, the developmental disorders were clinically obvious. However, in three of the patients whilst the attending clinicians suspected developmental delay, they responded to parental anxiety over pace of development; the Developmental Screening Inventory (DSI), previously validated in the country was administered. ${ }^{12}$ The three subjects were found to be developmentally delayed and included in the study.

\section{EEG Recording}

The EEG recording was done on each subject using 20-channel computerized Medelec® EEG machine; with scalp electrode placement in accordance to the 10-20 system of the International Federation on EEG. ${ }^{13}$ The subject scheduled for recording was seated in a comfortable position in the recording room devoid of environmental distraction as much as possible. For the very young subjects and / or those not skillful to sit unaided, they were carried by their caregivers (in most cases their mothers) on their laps or strapped to the back as typical of African cultural practice. The recording EEG technologist was blinded to the clinical diagnoses of the subjects.

Awake recording was done on each subject. Pre-recording instructions were given, aimed at minimizing artefacts in the recording. The cooperation of the very young subjects noticed to have developed test anxiety or fear was secured as much as possible. Naturally, EEG recording on children of these young age groups posed some practical difficulties. However, obvious body movement noticed during the EEG recording was indicated as artefacts on the tracing. Furthermore, the recording was usually suspended during such body movement; but on the whole the suspension time was noticed and added to make up to average 45 minutes recording. Where this could not be achieved (in three cases), there was need to sedate the subject by administering intravenous diazepam to carry out the recording. This is a limitation as this could affect the detection of EEG abnormalities in such recordings.

Each recording session lasted 40-50 minutes (average of 45 minutes) including 3-5 minutes of hyperventilation for subjects that could comprehend the $\mathrm{H}-\mathrm{V}$ instruction).

The EEG reporting was done independently by two consultant psychiatrists trained on EEG interpretation with inter-rater reliability of 0.89 . The reporting was based on the EEG classification by Luders and Noachter ${ }^{14}$; that is, normal or nonspecific EEG changes of abnormal slow background rhythm as well as intermittent or continuos generalized, laterized or regional slowing. Specific abnormalities included spikes, polyspikes, sharp waves, sharp-slow waves, spike-wave and polyspike-wave complexes.

\section{Data Analyses}

Frequency distributions were obtained and chi square statistics were used to examine statistical significance between categorical variables. Correlation coefficients were carried out to examine bivariate relationships between the variables. Associations and differences were said to be significant when the p- value was $\leq 0.05$. 


\section{Results}

\section{Socio-demographic Data}

A total of one hundred and eleven (1 1 l) subjects were evaluated for developmental disorders from the three centres during the 36month study period; made up of 63(56.8\%) males and 48(43.2\%) females. The highest age group was 0-5years (69.4\%), followed by 6 -10years (20.7\%) and 11-15years (9.9\%). The mean age was 4.8years ( \pm 3.9$)$.

\section{Diagnoses/Neuropsychiatric Complications}

In accordance with the International Classification of Diseases, 10th Edition (ICD 10), the clinical diagnoses on the subjects are as shown in Table I. It shows that the mixed specific developmental disorders (mainly admixture of speech and motor dysfunctions) constituted the highest (55\%), followed by specific developmental disorders of speech and language (34.2\%). Furthermore, forty-one subjects (36.9\%) suffered from notable complications, which included seizures, 22(19.8\%), Restlessness/Hyperactivity, 15(13.5\%) and psychotic symptoms, 4(3.6\%).

In the majority (76.6\%) of the subjects, the cause(s) of the developmental disorder could not be ascertained; while it was only in $23.4 \%$ that the cause(s) were apparent. Of these, the main putative aetiologies included severe seizure during neonatal period and birth asphyxia. In none of the subjects was there retrospective history of developmental delay and/or seizure in the parents while they were young. However, in one subject, there was history of developmental delay in one of the siblings; while for two of the subjects, there was history of seizure in one sibling of each of these two.

\section{EEG Findings}

The EEG was normal in 22 (19.8\%) of the subjects; while the rest, $89(80.2 \%)$ had one form of EEG abnormality or the other. The commonest abnormalities were the epileptiform activities of sharp waves, spikes/polyspikes and spike-wave complexes found in 85 (76.6\%) of the subjects; followed by non-specific slow activities in $4(3.6 \%)$.

On the localization of the EEG abnormalities; most were focal activities (51.4\%) with 40.5\% found over the fronto-temporal region, followed by temporal lobe (2.8\%)and frontal lobe (1.8\%).

Generalized paroxysmal (burst) activities were recorded in less than one-third (28.8\%) of cases. With regards to lateralization of the focal EEG abnormalities, 45\% were bilateral; distantly followed by right-sided focus (5.5\%) and left sided (0.9\%). Tables II shows EEG findings/ abnormalities in relation to clinical diagnoses on the subjects.

Table I: Distribution according to diagnoses and associated neuropsychiatric complications.

\begin{tabular}{|c|c|c|c|}
\hline \multirow{2}{*}{ DIAGNOSIS } & \multicolumn{3}{|c|}{ Frequency \& Percentage (\%) } \\
\hline & M & $F$ & Subtotal \\
\hline $\begin{array}{l}\text { SPECIFIC DEVELOPMENTAL DISORDER OF SPEECH AND LANGUAGE } \\
\text { i. With Restlessness/ Hyperactivity } 105 \\
\text { ii. With Seizure } 10 \\
\text { iii. With Psychotic Symptoms } 21 \\
\text { iv. Without Complication } 190\end{array}$ & $32(50.8)$ & $6(12.5)$ & $38(34.2)$ \\
\hline $\begin{array}{l}\text { SPECIFIC DEVELOPMENTAL DISORDE OF MOTOR FUNCTION } \\
\text { i. With Restlessness/ Hyperactivity } 00 \\
\text { ii. With Seizure } 50 \\
\text { iii. With Psychotic Symptoms } 00 \\
\text { iv. Without Complication } 05\end{array}$ & $5(7.9)$ & $5(10.4)$ & $10(9.0)$ \\
\hline $\begin{array}{l}\text { MIXED SPECIFIC DEVELOPMENTAL DISORDER } \\
\text { i.With Restlessness/ Hyperactivity } 00 \\
\text { ii. With Seizure } 016 \\
\text { iii. With Psychotic Symptoms } 10 \\
\text { iv. Without Complication } 2420\end{array}$ & 25(39.7) & $36(75)$ & $61(55.0)$ \\
\hline PERVASIVE DEVELOPMENTAL DISORDER & $1(1.6)$ & $1(2.1)$ & $2(1.8)$ \\
\hline TOTAL & $63(100)$ & $48(100)$ & $111(100)$ \\
\hline
\end{tabular}

Table II: Clinical diagnoses and eeg abnormalities/findings

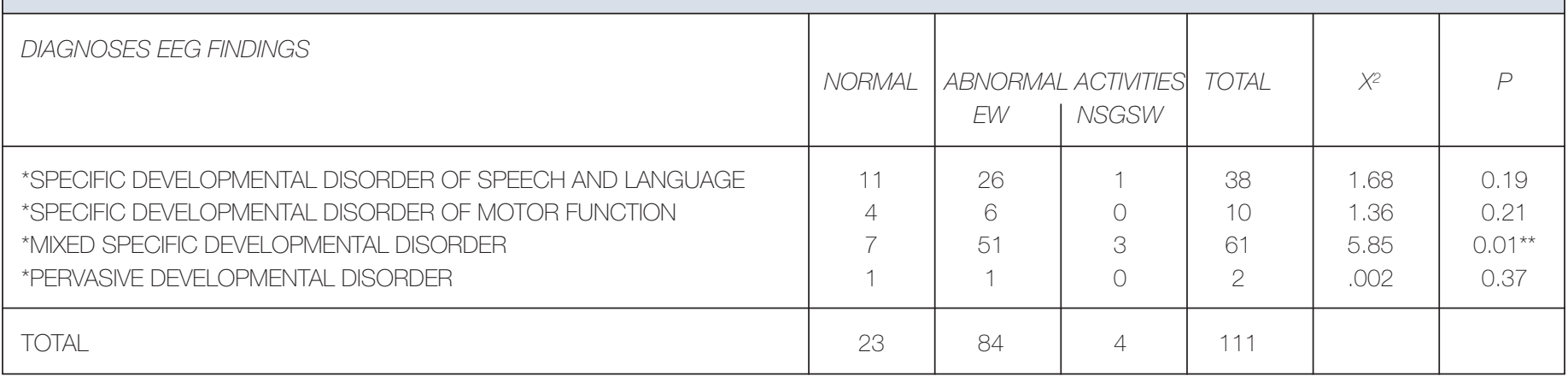


There was no statistically significant relationship between EEG abnormalities and clinical diagnoses except for mixed specific developmental disorder $(p=0.01)$. Table III shows EEG

abnormalities according to age of the subjects. It is shown from the table that there was no statistically significant relationship between EEG abnormalities and age.

\begin{tabular}{|l|c|c|c|c|c|c|}
\hline \multicolumn{6}{|c|}{ Table III: EEG abnormalities according to age group and sex } \\
\hline $\begin{array}{l}\text { Age Group } \\
\text { (years) }\end{array}$ & \multicolumn{2}{|c|}{ EEG FINDINGS } & & & \\
\hline Normal & $E W$ & NSGSW & TOTAL & $X 2$ & $P$ \\
-5 $5-10$ & 14 & 61 & 2 & 77 & 1.02 & 0.31 \\
$11-15$ & 2 & 21 & 0 & 23 & 0.03 & 1.00 \\
& 7 & 2 & 2 & 11 & 0.00 & 0.58 \\
\hline TOTAL & 23 & 84 & 4 & 111 & 14.35 & 0.0008 \\
\hline
\end{tabular}

NOTE: EW= Epileptiform Waves

NSGSW = None Specific Generalized Slow Waves.

\section{Correlation Analyses (Spearman Nonparametric Correlations)}

The EEG abnormalities correlated with the lateralization (.48**) and localization $\left(.47^{* *}\right)$ of these abnormalities. Lastly, the localization correlated strongly with lateralization (.86**) of the EEG abnormalities. The correlations are significant at the 0.01 levels. The variables of age, sex, diagnoses did not have significant correlation with EEG abnormalities, localization and lateralization.

\section{Discussion}

The preponderance of males over their female counterparts in this study is similar to findings on the prevalence of mental health problems, among children in developing countries. ${ }^{15,16}$

Although mixed specific developmental disorder is a residual diagnostic category, with an admixture of specific developmental disorders of speech and language, motor functions etc. but none of them predominating ${ }^{1}$, it constituted over slightly half (55\%) of the cohort in this study. Perhaps, the predominance of this diagnosis in our cohort could partly be explained by widespread malnutrition and infection among children in the country ${ }^{7,17}$; with attendant diffuse cerebral atrophy ${ }^{18}$ and subsequent poor development in various psychomotor fields..$^{19}$ Furthermore, of the specific developmental disorders, that of speech (34.2\%) is about four times more common in this study than the specific abnormality of motor function (9\%) which is in keeping with findings from previous studies. $2,4,5$ Also, pervasive developmental disorder, as in our study (1.8\%) still constitutes a rare condition. 8,11

The difficulty encountered in obtaining an exhaustive comprehensive clinical history for children with mental health problems in developing countries, such as Nigeria, was reflected in this study whereby the causative factor(s) of the developmental disorders could not be ascertained in over three-quarter (76.6\%) of the subjects. Similar difficulties have been noted from previous studies. ${ }^{15,16,20}$ In our study, of the remaining $23.4 \%$ subjects, the commonest aetiological factors included birth asphyxia and repeated / severe seizures during their neonatal periods. It is instructive to note that these problems can be largely ameliorated by improved maternal and child health care services. ${ }^{21,22}$

Subjects with developmental disorder are known to suffer from complications; examples of which include restlessness / hyperactivity, seizure, psychotic symptoms etc. In our study, 36.9\% of the subjects suffered from these complications, of which seizure (27\%) was the commonest. Previous studies have consistently shown seizure to be a major complication among children with developmental disorders. ${ }^{2,4}$ Consequently, and coupled with a possible association between abnormal EEG and developmental regression, some clinicians advocate an EEG recording as routine. 23,24

In the current study, the majority (79.3\%) of the cohort had one at least one form of EEG abnormality, of which epileptiform activities were the commonest (75.7\%). The high percentage of abnormalities in the subjects' EEG recordings could be due to subtle brain damage possibly causing developmental disorders and /or associated complications such as seizure among the cohort. These clinical conditions are known to be associated with a high level of EEG abnormalities. The finding of epileptiform activity being the commonest EEG abnormality is in agreement with previous findings in prolonged EEG recordings on such subjects ${ }^{23}$, even in the absence of clinical seizures. ${ }^{25}$ The presence of epileptiform activity on the EEG recordings of subjects without clinical seizures does not warrant treatment with anticonvulsants. However, for this category of subjects, they need to be monitored at clinic follow-up because of the risk of having seizures in later life. Epileptiform waves or activity, according to the International Federation of Societies for Electroencephalography and clinical Neurophysiology (IFSECN) have been defined as "distinctive waves or complexes, distinguished from background activity, and resembling those recorded in a proportion of human subjects suffering from epileptic disorders"'. ${ }^{13}$ Such activity includes sharp waves, spikes/polyspikes, spike-wave complexes and polyspikewave complexes; but these morphological distinctions are less important than the certainty of their presence. ${ }^{26}$ A higher percentage of our cohort (79.3\%) had EEG abnormalities compared with studies from the Western world with findings of 50\% even among individuals with autism and pervasive developmental disorders. ${ }^{27}$ Perhaps, this could be explained by the fact that African children are reported to be at higher risk of experiencing brain damage early in life when compared to their Caucasian counterparts. ${ }^{28}$

With respect to the localization of focal epileptiform activity, over $40 \%$ of such EEG abnormalities among the cohort were in the fronto-temporal region. This finding is in agreement with those from previous studies where the temporal and frontal lobes were the commonest regions for focal EEG abnormalities. ${ }^{26,29}$ From Table II, it is seen that subjects with mixed specific developmental disorder had the greatest percentage of epileptiform activity i.e 51 out of 61 (83.6\%); this compared to specific developmental disorder of speech and language (68.4\% i.e 26 out of 38) and that of motor function $(60 \%, 6$ out of 10). It is possible that subjects with the mixed specific developmental disorder could have had more extensive brain damage so as to increase the risk of having epileptiform activity on their EEG recordings. From Table III, epileptiform activity was more common on the EEG tracings of the younger age groups of $0-5$ years (79.2\%) and 6- 10 years (91.3\%); when compared with that of 11-15 years (18.1\%). This is similar to findings from previous studies where EEG abnormalities are inversely related to age. 23,26

In the current study, we found significant correlations between EEG abnormalities and localization as well as lateralization of such abnormalities. This indicates an agreement with the earlier mentioned fact of the strong relationship between focal EEG 
abnormalities and lateralization as well as localization.

It should be noted that there was difficulty at detecting the possible presence of absence attacks and subtle complex-partial seizures especially in the very young aged subjects. In addition, brain imaging could not be done to complement the EEG findings. Brain imaging investigations are very expensive and cost on the average 500 to 800 dollars per person in Nigeria.

\section{Conclusion}

It is concluded that because of their chronicity and attendant health care / educational burden, there is need to tackle the problem of developmental abnormalities in Nigeria with greater attention being paid to preventive health care within maternal and child medical services.

\section{Acknowledgement}

The authors are grateful to Dr. O. A. Owoeye (one of the EEG iterpreters), the entire staff of EEG unit, Psychiatric Hospital, Yaba, Lagos and Mr Isiaka G Amoo for his secretarial assistance.

\section{References}

1. World Health Organization. The ICD-10 classification of Mental and Behavioural disorders: Clinical descriptions and diagnotic Guidlines. Oxford University Press. Walton Street, Oxford, 1992

2. Knobloch $H$ and Pasamanick B (ed). Problems of differential diagnosis. In Gesell and Amatruda's Developmental diagnosis 3rd edition. Harper and Row: Hagerstown M. D. 1974; pp 129 - 148.

3. http://www.firstsigns.org/delays_disorder/delays_disorders.htm. Developmental and Behavioural Disorders. Assessed on 18 May 2005.

4. Gelder M; Mayou R and Geddes J. (eds) Disorders of Development In Oxford Core Texts. Psychiatry (2nd Edition). Oxford University Press 1999: pp $414-418$

5. Parker S. and Zuckerman B. (eds) Behavioural and Developmental Pediatrics. A handbook for Primary Care. Little, Brown and Company. 1995.

6. Olatawura $M O$, Odejide $A O$. Child psychiatric disorders in Ibadan. Nigerian Journal of Paediatrics 1976; 3:9-14.

7. Remington J S, McLeod R, Thulliez P, Desmonts G. Toxoplasmosis, In Infectious diseases of the fetus and newborn infant. Edited by Remington JS, Klein JO. Philadelphia, WB Saunders, 2001: pp 205 - 346.

8. Palmer RF, Blanchard S, Jean CR, Mandell DS. School district resources and identification of Children with autistic Disorder. American Journal of Public Health 2005; 95; 1: $125-130$.

9. Pasamanick B. Patterns of research in mental health hygiene. Psychiatric Quarterly 1952; 26: 577 - 589.

10. Buka SL, Tsuang MT, Torrey EF, Klebanoff MA, Bernstein D, Yolken RH. Maternal infectious and subsequent psychosis among offsprings. Archives of General Psychiatry 2001, 58: 1032 - 1037.

11. Binnie CD, Boyd S. Clinical Neurophysiology. In M Rutter, E Taylor and L
Hersov (eds). Child and Adolescent Psychiatry. Modern Approaches. Third Edition. Blackwell Science 1994: pp 110 - 126

12. Aina OF, Morakinyo O. The validation of Developmental Screening Inventory (DSI) on Nigerian Children. Journal of Tropical Paediatrics 2001; 47: $323-328$

13. International Federation of Societies for Electroencephalography and Clinical Neurophysiology (IFSECN). A glossary of terms most commonly used by clinical Electroencephalographers, Electroencephalography and Clinical Neurophysiology 1974; 37: 538 - 548.

14. Luders HO, Noachter SS. Atlas and Classification of Electroencephalography. Philadelphia: WB Saunders, 2000

15. Giel R, DeArango MV, Climent CE, Harding TW et al. Childhood Mental Disorders in Primary Health Care: Results of Observation in Four Developing Countries. Paediatrics 1981; 68: 677 - 683.

16. Omigbodun OO. Psychosocial issues in a Child and Adolescent psychiatric clinic. Social Psychiatry and Psychiatric Epidemiology 2004; 39: $667-672$

17. Aina OF, Morakinyo O. Anthropometric Assessment in Nigerian Children. East African Medical Journal 2001; 78; 6: 33 - 37.

18. Houscham KC, DeVilliers JFK. Computed Tomography in Severe protein energy malnutrition. Archives of disease in childhood 1987; 62: 589 - 592.

19. Galler JR, Ramsey F, Forde V. A follow-up study of the influence of early malnutrition on subsequent development, IV: intellectual performance during adolescence. Nutrition and Behaviour 1986; 3: 211 -222.

20. Ohaeri JU, Odejide AO, Gureje O, Olatawura MO. Psychiatric Morbidity among Children Attending Health facilities in Primary Health Care in a Rural Nigerian Community. Nigerian Medical Journal 1994; 26: 93 - 96.

21. Integrated Management of Pregnancy and Childbirth (IMPAC). Geneva: WHO Reproductive Health and Research, 2000.

22. Sharma M, Uprety D, Pokhrel M, Karki A, Sharma U, Babu S. Maternal mortality of BP Koirala Institute of Health Sciences, Nepal: review of 6 years. Tropical Doctor 2005; 35; 1: 25 - 26.

23. Tuchman RI, Rapin I. Regression in pervasive developmental disorders: Seizures and epileptiform electroencephalogram correlates. Pediatrics 1997; 99 (4): 560 - 566

24. Wing L. The autistic spectrum. Lancet 1997; 350: 1761 - 1766.

25. American Psychiatric Association. Usually first diagnosed in Infancy, Childhood or Adolescence In Diagnosis and Statistical Manual of Mental Disorders: DSM IV. Washington; American Psychiatric Association, 1994

26. Bromfield EB. Epileptiform Discharges. http://www.emedicine.com/neuro/topicl28.htm (last updated July 8, 2002). Assessed 9/3/2005.

27. Prosser JGS, Le Couteur AS. Autism and the pervasive developmental disorders. Current Paediatrics 1997; 7: 158 - 162.

28. Asuni T, Schoenberg F, Swift C. Mental Health and disease in Africa (eds). Spectrum Books Ltd, Ibadan 1994.

29. Shewmon DA, Erwin RJ. Focal spike-induced cerebral dysfunction is related to the after slow wave. Annals of neurology 1988; 23 (2): 131 137. 